PROCEEDINGS OF THE

AMERICAN MATHEMATICAL SOCIETY

Volume 129, Number 9, Pages 2753-2762

S 0002-9939(01)05828-2

Article electronically published on January 23, 2001

\title{
UNIQUENESS OF LEFT INVARIANT SYMPLECTIC STRUCTURES ON THE AFFINE LIE GROUP
}

\author{
YOSHIO AGAOKA
}

(Communicated by Jozef Dodziuk)

\begin{abstract}
We show the uniqueness of left invariant symplectic structures on the affine Lie group $A(n, \mathbf{R})$ under the adjoint action of $A(n, \mathbf{R})$, by giving an explicit formula of the Pfaffian of the skew symmetric matrix naturally associated with $A(n, \mathbf{R})$, and also by giving an unexpected identity on it which relates two left invariant symplectic structures. As an application of this result, we classify maximum rank left invariant Poisson structures on the simple Lie groups $S L(n, \mathbf{R})$ and $S L(n, \mathbf{C})$. This result is a generalization of Stolin's classification of constant solutions of the classical Yang-Baxter equation for $\mathfrak{s l}(2, \mathbf{C})$ and $\mathfrak{s l}(3, \mathbf{C})$.
\end{abstract}

\section{INTRODUCTION}

The purpose of this paper is to show the uniqueness of left invariant symplectic structures on the affine Lie group $A(n, \mathbf{R})$, and as its application, to classify maximum rank constant solutions of the classical Yang-Baxter equation for two simple Lie groups $S L(n, \mathbf{R})$ and $S L(n, \mathbf{C})$, which gives a partial answer to the question posed by Liu and $\mathrm{Xu}$ [7, p.36].

It is well known that the even dimensional Lie group $A(n, \mathbf{R})$ defined by

$$
A(n, \mathbf{R})=\left\{\left(\begin{array}{cc}
A & v \\
0 & 1
\end{array}\right) \mid \begin{array}{l}
A \in G L(n, \mathbf{R}) \\
v \in \mathbf{R}^{n}
\end{array}\right\}
$$

admits a left invariant symplectic structure, and its structure is described by several types of symplectic forms (cf. 2], 3], 8, 3], 10]). In this paper, we first prove that they are essentially equivalent. More precisely, we show that any left invariant symplectic structure on $A(n, \mathbf{R})$ is equivalent to any other such structure under the adjoint action of $A(n, \mathbf{R})$.

For general Lie groups $G$, it is well known that the exact 2-form $d \alpha\left(\alpha \in \mathfrak{g}^{*}\right)$ gives a left invariant symplectic structure on $G$ if and only if the coadjoint orbit of $\alpha$ is open in $\mathfrak{g}^{*}$ (cf. 88). This fact implies that, in the case $H^{2}(\mathfrak{g}, \mathbf{R})=0$, left invariant symplectic structures on $G$ (provided they exist) are locally rigid under the adjoint action. But it does not imply the global rigidity (= uniqueness) because there may

Received by the editors January 12, 2000 and, in revised form, January 18, 2000.

2000 Mathematics Subject Classification. Primary 53C30, 53D05, 53D17; Secondary 17B99.

Key words and phrases. Left invariant symplectic structure, affine Lie group, Pfaffian, Poisson structure, classical Yang-Baxter equation. 
exist a different type of symplectic structure situated outside of the coadjoint orbit of $d \alpha$. And to show the global rigidity, we need a more delicate algebraic device fitted to the group structure of $G$, which is in general quite difficult to find.

In the case of $A(n, \mathbf{R})$, we already know the vanishing of the second cohomology $H^{2}(\mathfrak{a}(n, \mathbf{R}), \mathbf{R})$, where $\mathfrak{a}(n, \mathbf{R})$ is the Lie algebra of $A(n, \mathbf{R})$, and hence, any left invariant symplectic structure on $A(n, \mathbf{R})$ is exact. For this group, we prove the global rigidity by showing unexpected identities concerning the Pfaffian of the skew symmetric matrix naturally associated with $\mathfrak{a}(n, \mathbf{R})$ (Lemma 2). Precisely, we show that in terms of these identities, the non-degeneracy of the exact 2-form $d \alpha$ is equivalent to the non-degeneracy of a certain matrix $g_{\alpha}$ which is canonically determined by $\alpha \in \mathfrak{a}(n, \mathbf{R})^{*}$. In addition, we show that the coadjoint action of this matrix $g_{\alpha} \in A(n, \mathbf{R})$ transforms the exact symplectic form $d \alpha$ to the standard one which we fixed in advance. And these facts imply the uniqueness of left invariant symplectic structure on $A(n, \mathbf{R})$. To construct the matrix $g_{\alpha}$, we use matrices appearing in Cayley-Hamilton type theorem.

As an application of this result, in the second half of this paper, we classify maximum rank left invariant Poisson structures on the Lie groups $S L(n, \mathbf{R})$ and $S L(n, \mathbf{C})$ (cf. 1], 2, 13]). It is known that left invariant Poisson structures on a Lie group $G$ correspond to constant solutions of the classical Yang-Baxter equation, which also correspond to the pairs consisting of Lie subalgebras of $\mathfrak{g}$ and non-degenerate 2-cocycles of them (cf. Proposition 4). In this standpoint, Belavin and Drinfel'd [2] gave an example of a constant solution of the classical Yang-Baxter equation for $S L(n, \mathbf{C})$ and $G L(n, \mathbf{C})$ with rank $=n(n-1)$. But the complete classification of solutions for these groups seems hopeless as stated in [2. p.179] because there are too many degenerate low rank solutions. In this situation, it is a natural and fundamental problem to determine which is the most generic (= highest rank) solution of the classical Yang-Baxter equation. The second main purpose of this paper is to give the answer to this question for two simple Lie groups $S L(n, \mathbf{R})$ and $S L(n, \mathbf{C})$.

We first show that the maximum rank of solutions of the classical Yang-Baxter equation is $n(n-1)$, namely, the solution constructed in [2] is of maximum rank. We prove this fact by using Dynkin's classification of subgroups of classical Lie groups [6]. Next we classify such maximum rank solutions under the action of the adjoint groups or more generally the action of the automorphism groups of Lie algebras. The results for $S L(n, \mathbf{R})$ and $S L(n, \mathbf{C})$ are summarized in Theorem 3 and Theorem 6, respectively. In particular, for both groups, the maximum rank solution is uniquely determined under the action of the automorphism groups of Lie algebras. This result for $S L(n, \mathbf{C})$ may be considered as a generalization of Stolin's classification of constant solutions of the classical Yang-Baxter equation for $\mathfrak{s l}(2, \mathbf{C})$ and $\mathfrak{s l}(3, \mathbf{C})$. To prove these theorems, we use the facts that the maximum dimensional proper subalgebra of $\mathfrak{s l}(n, \mathbf{R})$ is isomorphic to $\mathfrak{a}(n-1, \mathbf{R})$ (Proposition 5 ), and the uniqueness of left invariant symplectic structures on it which we prove in section 2 .

For other simple Lie groups, the examples of high rank constant solutions of the classical Yang-Baxter equation were known only for a few cases. Concerning this problem, in the paper [1], we constructed a non-trivial high rank solution of the classical Yang-Baxter equation for each classical non-compact simple Lie group. And as a next problem, it is desirable to decide whether the global rigidity also holds for these groups, as in the case of $S L(n, \mathbf{R})$ or $S L(n, \mathbf{C})$. 


\section{Left inVARIant SympleCtic StruCtures on $A(n, \mathbf{R})$}

Let $A(n, \mathbf{R})$ be the affine Lie group defined in Introduction. The first purpose of this paper is to show the following theorem.

Theorem 1. The Lie group $A(n, \mathbf{R})$ admits a unique left invariant symplectic structure under the adjoint action of $A(n, \mathbf{R})$.

We first explain the explicit construction of a left invariant symplectic structure on $A(n, \mathbf{R})$, following [2]. The Lie algebra of $A(n, \mathbf{R})$ is expressed as

$$
\mathfrak{a}(n, \mathbf{R})=\left\{\left(\begin{array}{cc}
A & v \\
0 & 0
\end{array}\right) \mid \begin{array}{l}
A \in \mathfrak{g} \mathfrak{l}(n, \mathbf{R}) \\
v \in \mathbf{R}^{n}
\end{array}\right\}
$$

and it is known that the second cohomology group $H^{2}(\mathfrak{a}(n, \mathbf{R}), \mathbf{R})$ vanishes (cf. [3], [8], 9]). Hence, any left invariant symplectic structure on $A(n, \mathbf{R})$ is exact. Let $E_{i j}$ be the matrix such that the $(i, j)$-component is 1 and other components are all zero. Then, $\left\{E_{i j}\right\}_{1 \leq i \leq n, 1 \leq j \leq n+1}$ forms a basis of $\mathfrak{a}(n, \mathbf{R})$. We denote by $\left\{E_{i j}^{*}\right\}$ its dual basis, and put

$$
\alpha_{0}=E_{12}^{*}+E_{23}^{*}+\cdots+E_{n, n+1}^{*}, \quad \omega=-d \alpha_{0},
$$

i.e., $\omega(X, Y)=\alpha_{0}([X, Y])$ for $X, Y \in \mathfrak{a}(n, \mathbf{R})$. Then, the 2-form $\omega$ is nondegenerate and gives a left invariant symplectic structure on $A(n, \mathbf{R})$ (cf. 2]; this fact can also be verified by using Lemma 2 (1) below). As we stated above, any left invariant symplectic structure on $A(n, \mathbf{R})$ is exact, and hence to prove Theorem 1 , we only need to show the following fact: If $-d \alpha$ is non-degenerate for $\alpha \in \mathfrak{a}(n, \mathbf{R})^{*}$, then there exists an element $g \in A(n, \mathbf{R})$ such that $(\operatorname{Ad} g)^{*} \alpha_{0}=\alpha$, where $(\operatorname{Ad} g)^{*}: \mathfrak{a}(n, \mathbf{R})^{*} \rightarrow \mathfrak{a}(n, \mathbf{R})^{*}$ is the dual map of Ad $g: \mathfrak{a}(n, \mathbf{R}) \rightarrow \mathfrak{a}(n, \mathbf{R})$. (In the following, we often say that $\alpha \in \mathfrak{a}(n, \mathbf{R})^{*}$ is non-degenerate in case $-d \alpha$ is non-degenerate.)

To prove the above fact, we first define the polynomials depending on an $(n, n)$ matrix $A$ as follows. Let $\left\{\varepsilon_{1}, \cdots, \varepsilon_{n}\right\}$ be the eigenvalues of a matrix $A$, and put

$$
f_{k}(A)=(-1)^{k} \sum_{i_{1}<\cdots<i_{k}} \varepsilon_{i_{1}} \cdots \varepsilon_{i_{k}}
$$

for $0 \leq k \leq n$. For example, we have

$$
\begin{aligned}
f_{0}(A) & =1 \\
f_{1}(A) & =-\operatorname{Tr} A \\
f_{2}(A) & =1 / 2 \cdot\left\{(\operatorname{Tr} A)^{2}-\operatorname{Tr}\left(A^{2}\right)\right\} \\
f_{3}(A) & =-1 / 6 \cdot\left\{(\operatorname{Tr} A)^{3}-3 \operatorname{Tr} A \cdot \operatorname{Tr}\left(A^{2}\right)+2 \operatorname{Tr}\left(A^{3}\right)\right\} \\
& \cdots \cdots \cdots \\
f_{n}(A) & =(-1)^{n} \operatorname{det} A .
\end{aligned}
$$

Note that $f_{k}(A)$ is invariant under the adjoint action of $G L(n, \mathbf{R})$. Next, we put

$$
\varphi_{k}(A)=A^{k-1}+f_{1}(A) A^{k-2}+f_{2}(A) A^{k-3}+\cdots+f_{k-1}(A) I_{n}
$$

for $1 \leq k \leq n+1$. Clearly, from Cayley-Hamilton's theorem, we have $\varphi_{n+1}(A)=0$.

Now, we express $\alpha \in \mathfrak{a}(n, \mathbf{R})^{*}$ as

$$
\alpha=\sum_{1 \leq i, j \leq n} a_{i j} E_{i j}^{*}+\sum_{1 \leq k \leq n} b_{k} E_{k, n+1}^{*},
$$


and define the $(n+1, n+1)$-matrix $g_{\alpha}$ by

$$
g_{\alpha}={ }^{t}\left(\begin{array}{ccccc}
\varphi_{n}(A) b & \cdots & \varphi_{2}(A) b & \varphi_{1}(A) b & 0 \\
f_{n}(A) & \cdots & f_{2}(A) & f_{1}(A) & 1
\end{array}\right),
$$

where $A=\left(a_{i j}\right)$ and $b={ }^{t}\left(b_{1} \cdots b_{n}\right)$. We rearrange the basis of $\mathfrak{a}(n, \mathbf{R})$ as

$$
\begin{array}{lcl}
X_{1}=E_{11}, & \cdots & X_{n}=E_{1 n}, \\
X_{n+1}=E_{21}, & \ldots & X_{2 n}=E_{2 n}, \\
& \cdots \cdots & \\
X_{n^{2}-n+1}=E_{n 1}, & \cdots & X_{n^{2}}=E_{n n}, \\
X_{n^{2}+1}=E_{1, n+1}, & \cdots & X_{n(n+1)}=E_{n, n+1} .
\end{array}
$$

Then, the following key lemma holds. Theorem 1 follows immediately from this lemma.

Lemma 2. (1) The Pfaffian of the skew symmetric $(n(n+1), n(n+1))$-matrix $\left(\alpha\left(\left[X_{i}, X_{j}\right]\right)\right)$ is equal to $\left|g_{\alpha}\right|$. In particular, $-d \alpha$ is non-degenerate if and only if the matrix $g_{\alpha}$ is non-singular.

(2) If $g_{\alpha}$ is non-singular, then $g_{\alpha} \in A(n, \mathbf{R})$ and the equality $\left(\operatorname{Ad} g_{\alpha}\right)^{*} \alpha_{0}=\alpha$ holds.

Proof. (1) We show the equality

$$
\operatorname{det}\left(\alpha\left(\left[X_{i}, X_{j}\right]\right)\right)=\left|\varphi_{n}(A) b \cdots \varphi_{2}(A) b \varphi_{1}(A) b\right|^{2} .
$$

To prove (\#), we consider the complexified version of this equality, i.e., we assume that $\alpha \in \mathfrak{a}(n, \mathbf{C})^{*}, A \in \mathfrak{g l}(n, \mathbf{C})$ and $b \in \mathbf{C}^{n}$. Under this situation, we consider the coadjoint action $(\operatorname{Ad} g)^{*}(g \in G L(n, \mathbf{C}) \subset A(n, \mathbf{C}))$ on both sides of the equality (\#). If $\alpha \in \mathfrak{a}(n, \mathbf{C})^{*}$ corresponds to the pair $(A, b) \in \mathfrak{g l}(n, \mathbf{C}) \oplus \mathbf{C}^{n}$, then the element $(\operatorname{Ad~} g)^{*} \alpha$ corresponds to the pair $\left({ }^{t} g A^{t} g^{-1},{ }^{t} g b\right)$. Hence, by this action, the right-hand side of the equality (\#) is multiplied by $|g|^{2}$ because $\varphi_{k}\left({ }^{t} g A^{t} g^{-1}\right)$ $={ }^{t} g \varphi_{k}(A)^{t} g^{-1}$.

On the other hand, by the same action, the matrix $\left(\alpha\left(\left[X_{i}, X_{j}\right]\right)\right)$ is transformed into $^{t}(\operatorname{Ad} g)\left(\alpha\left(\left[X_{i}, X_{j}\right]\right)\right)(\operatorname{Ad} g)$. Hence, the left-hand side is multiplied by $|\operatorname{Ad} g|^{2}$, where Ad $g: \mathfrak{a}(n, \mathbf{C}) \rightarrow \mathfrak{a}(n, \mathbf{C})$. The map Ad $g$ preserves both subspaces $\mathfrak{g l}(n, \mathbf{C})$ and $\mathbf{C}^{n}$ in $\mathfrak{a}(n, \mathbf{C})$, and it is easy to see that

$$
\operatorname{det}\left(\left.\operatorname{Ad} g\right|_{\mathfrak{g r}(n, \mathbf{C})}\right)=1, \quad \operatorname{det}\left(\left.\operatorname{Ad} g\right|_{\mathbf{C}^{n}}\right)=|g| .
$$

Hence, both sides of the equality (\#) are multiplied by $|g|^{2}$ under the action of $(\operatorname{Ad} g)^{*}$.

Then, since generic elements $A \in \mathfrak{g l}(n, \mathbf{C})$ are transformed into diagonal matrices by an adjoint action of $G L(n, \mathbf{C})$, we only need to show the above equality (\#) in the case $A$ is a diagonal matrix. Express $A=\operatorname{diag}\left(a_{1} \cdots a_{n}\right)$, and we show that both sides are equal to

$$
\left\{b_{1} \cdots b_{n} \prod_{i<j}\left(a_{i}-a_{j}\right)\right\}^{2} .
$$

We first compute the determinant of the skew symmetric $(n(n+1), n(n+1))$ matrix $\left(\alpha\left(\left[X_{i}, X_{j}\right]\right)\right)$. For this purpose, we only need to consider the bracket operations of $\mathfrak{a}(n, \mathbf{C})$ which take values in the subspace $\left\langle E_{i i}, E_{j, n+1}\right\rangle \subset \mathfrak{a}(n, \mathbf{C})$ because $A$ is diagonal. These are essentially exhausted by

$$
\left[E_{i j}, E_{j i}\right]=E_{i i}-E_{j j}(i \neq j), \quad\left[E_{i j}, E_{j, n+1}\right]=E_{i, n+1},
$$


and by using this fact, the matrix $\left(\alpha\left(\left[X_{i}, X_{j}\right]\right)\right)$ is symbolically expressed as

\begin{tabular}{c|cccccc} 
& $E_{i j}$ & $E_{j i}$ & $E_{i i}$ & $E_{j j}$ & $E_{i, n+1}$ & $E_{j, n+1}$ \\
\hline$E_{i j}$ & 0 & $a_{i}-a_{j}$ & 0 & 0 & 0 & $b_{i}$ \\
$E_{j i}$ & $a_{j}-a_{i}$ & 0 & 0 & 0 & $b_{j}$ & 0 \\
$E_{i i}$ & 0 & 0 & 0 & 0 & $b_{i}$ & 0 \\
$E_{j j}$ & 0 & 0 & 0 & 0 & 0 & $b_{j}$ \\
$E_{i, n+1}$ & 0 & $-b_{j}$ & $-b_{i}$ & 0 & 0 & 0 \\
$E_{j, n+1}$ & $-b_{i}$ & 0 & 0 & $-b_{j}$ & 0 & 0
\end{tabular}.

Hence, by considering this table, the determinant of $\left(\alpha\left(\left[X_{i}, X_{j}\right]\right)\right)$ is equal to

$$
b_{1}^{2} \cdots b_{n}^{2} \operatorname{det}\left(\alpha\left(\left[E_{i j}, E_{j i}\right]\right)\right)_{i \neq j}=\left\{b_{1} \cdots b_{n} \prod_{i<j}\left(a_{i}-a_{j}\right)\right\}^{2} .
$$

Next, for the right-hand side, we have

$$
\left|\varphi_{n}(A) b \cdots \varphi_{2}(A) b \varphi_{1}(A) b\right|=\left|A^{n-1} b \cdots A b \quad b\right|
$$

for any $\alpha$, and from Vandermonde's formula, this value equals $b_{1} \cdots b_{n} \prod_{i<j}\left(a_{i}-a_{j}\right)$ if $A$ is diagonal. Therefore, the desired equality in Lemma 2 (1) holds.

(2) Assume $g_{\alpha}$ is non-singular. Then, the right multiplication $R_{g_{\alpha}}: \mathfrak{a}(n, \mathbf{R}) \rightarrow$ $\mathfrak{a}(n, \mathbf{R})$ gives a linear isomorphism. Hence, we only need to prove the equality for matrices of the form $\left(\begin{array}{cc}X & v \\ 0 & 0\end{array}\right) g_{\alpha}\left(X \in \mathfrak{g l}(n, \mathbf{R}), v \in \mathbf{R}^{n}\right)$. Since

$$
\operatorname{Ad} g_{\alpha}\left(\left(\begin{array}{cc}
X & v \\
0 & 0
\end{array}\right) g_{\alpha}\right)=g_{\alpha}\left(\begin{array}{cc}
X & v \\
0 & 0
\end{array}\right)
$$

it suffices to show the equality

$$
(\# \#) \quad\left\langle E_{12}^{*}+\cdots+E_{n, n+1}^{*}, g_{\alpha}\left(\begin{array}{cc}
X & v \\
0 & 0
\end{array}\right)\right\rangle=\left\langle\alpha,\left(\begin{array}{cc}
X & v \\
0 & 0
\end{array}\right) g_{\alpha}\right\rangle
$$

for $X \in \mathfrak{g l}(n, \mathbf{R})$ and $v \in \mathbf{R}^{n}$.

We first consider the case $X=E_{i j}$ and $v=0$. In this case, we have

$$
g_{\alpha}\left(\begin{array}{cc}
E_{i j} & 0 \\
0 & 0
\end{array}\right)=\left(\begin{array}{cc}
K & 0 \\
0 & 0
\end{array}\right)
$$

where $K$ is a matrix such that the $j$-th column is equal to the transpose of the $i$-th row of the matrix $\left(\varphi_{n}(A) b \cdots \varphi_{1}(A) b\right)$, and other columns are all zero. Hence, the left-hand side of the equality $(\# \#)$ is equal to the $i$-th component of the vector $\varphi_{n-j+2}(A) b$. On the other hand, the $i$-th row of the matrix $\left(\begin{array}{cc}E_{i j} & 0 \\ 0 & 0\end{array}\right) g_{\alpha}$ is equal to $\left({ }^{t} b^{t} \varphi_{n-j+1}(A) f_{n-j+1}(A)\right)$ and other rows of this matrix are all zero. Hence, the right-hand side of $(\# \#)$ is equal to

$$
\begin{aligned}
& \sum_{k} a_{i k} \times\left\{\text { the } k \text {-th component of } \varphi_{n-j+1}(A) b\right\}+b_{i} f_{n-j+1}(A) \\
= & \left\{\text { the } i \text {-th component of } A \varphi_{n-j+1}(A) b\right\}+f_{n-j+1}(A) b_{i} .
\end{aligned}
$$

But, from the definition of $\varphi_{k}(A)$, we have clearly

$$
\begin{aligned}
\varphi_{n-j+2}(A) b & =\left\{A \varphi_{n-j+1}(A)+f_{n-j+1}(A) I_{n}\right\} b \\
& =A \varphi_{n-j+1}(A) b+f_{n-j+1}(A) b .
\end{aligned}
$$

Therefore, both sides of the equality $(\# \#)$ take the same value. 
Next, we consider the case $X=0$ and $v=e_{i}$, where $\left\{e_{1}, \cdots, e_{n}\right\}$ is the canonical basis of $\mathbf{R}^{n}$. In this case, it is easy to check that both sides are equal to the $i$-th component of the vector $b$, and this completes the proof of Lemma 2 (2).

Remark. (1) From this lemma, we know that non-degenerate 1-forms $\alpha$ form an open dense orbit in $\mathfrak{a}(n, \mathbf{R})^{*}$, which implies that the pair $\left(A(n, \mathbf{R}), \mathfrak{a}(n, \mathbf{R})^{*}\right)$ is a (reducible) prehomogeneous vector space (cf. [12]).

(2) We can easily show that the element $g \in A(n, \mathbf{R})$ satisfying $(\operatorname{Ad} g)^{*} \alpha_{0}=\alpha$ is uniquely determined for each non-degenerate $\alpha$. In fact, if $g \in A(n, \mathbf{R})$ satisfies the equality $(\operatorname{Ad} g)^{*} \alpha_{0}=\alpha_{0}$, then by the same method stated at the beginning of the proof (2), we can show that $g=I_{n+1}$. We use this fact in section 3 .

(3) In [3], Bordemann, Medina and Ouadfel gave several conditions on $\alpha$ such that $-d \alpha$ is non-degenerate. From the above proof, it follows that $-d \alpha$ is nondegenerate if and only if

$$
\left|\begin{array}{llll}
A^{n-1} b & A^{n-2} b & \cdots & b
\end{array}\right| \neq 0
$$

and this condition just coincides with the second condition of Theorem 2.5 in 3 , p.429]. Clearly, the equality

$$
\left|\begin{array}{llll}
A^{n-1} b & A^{n-2} b & \cdots & b
\end{array}\right|=0
$$

gives the defining equation of the algebraic set consisting of degenerate $\alpha$ 's. Nondegeneracy of $\alpha_{0}=E_{12}^{*}+\cdots+E_{n, n+1}^{*}$ follows immediately from this fact. In addition, from the above proof, the 1 -form defined by

$$
\alpha=\sum a_{i} E_{i i}^{*}+\sum b_{j} E_{j, n+1}^{*}
$$

gives a left invariant symplectic structure $-d \alpha$ on $A(n, \mathbf{R})$ if and only if $a_{i} \neq a_{j}$ $(i \neq j)$ and $b_{j} \neq 0$. This example is nothing but the one stated in [3, p.430-431].

(4) It is desirable to show the equality in Lemma 2 (1) by purely algebraic methods, not depending on the topological argument. But unfortunately, we do not know this type of proof at present.

\section{Left invariant Poisson structures on $S L(n, \mathbf{R})$ and $S L(n, \mathbf{C})$}

As an application of Theorem 1, we prove the following theorem.

Theorem 3. Assume $n \geq 2$. Then, the maximum rank of left invariant Poisson structures on the Lie group $S L(n, \mathbf{R})$ is $n(n-1)$, and under the adjoint action of $S L(n, \mathbf{R})$, the number of equivalence classes of maximum rank left invariant Poisson structures on $S L(n, \mathbf{R})$ is

$$
\begin{cases}2 & (n=2 \text { or } n=\text { odd } \geq 3) \\ 4 & (n=\text { even } \geq 4)\end{cases}
$$

These Poisson structures are mapped to each other by the automorphism group of the Lie algebra $\mathfrak{s l}(n, \mathbf{R})$.

Before the proof, we briefly review some definitions and properties on left invariant Poisson structures on a general Lie group $G$ with Lie algebra $\mathfrak{g}$. For details, see 14], [1. A left invariant Poisson structure on $G$ is defined by the left translation of a 2-vector $\pi \in \wedge^{2} \mathfrak{g}$ which satisfies the classical Yang-Baxter equation $[\pi, \pi]_{S}=0$, where [, ] ${ }_{S}: \wedge^{2} \mathfrak{g} \times \wedge^{2} \mathfrak{g} \rightarrow \wedge^{3} \mathfrak{g}$ is the Schouten bracket defined by

$[X \wedge Y, Z \wedge W]_{S}=[X, Z] \wedge Y \wedge W+X \wedge[Y, Z] \wedge W-[X, W] \wedge Y \wedge Z-X \wedge[Y, W] \wedge Z$ 
$(X, Y, Z, W \in \mathfrak{g})$. The rank of the Poisson structure means the usual rank of a 2-vector $\pi \in \wedge^{2} \mathfrak{g}$. A left invariant Poisson structure can be also described in the following dual symplectic form (cf. [4):

Proposition 4. There is a one-to-one correspondence between the set of left invariant Poisson structures $\pi$ on a Lie group $G$ and the set of pairs $\left(\mathfrak{g}^{\prime}, \omega\right)$ where $\mathfrak{g}^{\prime}$ is an even dimensional subalgebra of $\mathfrak{g}$ and $\omega$ is a non-degenerate 2 -cocycle of $\mathfrak{g}^{\prime}$. Under this correspondence, the equality rank $\pi=\operatorname{dim} \mathfrak{g}^{\prime}$ holds.

For the explicit correspondence, see [4], 1]. We can easily see that this correspondence is Ad $G$-equivariant. In the following, we divide the proof of Theorem 3 into two steps. First, we classify maximum dimensional proper subalgebras of $\mathfrak{s l}(n, \mathbf{R})$ under the adjoint action of $S L(n, \mathbf{R})$, and next, classify non-degenerate 2 -cocycles (= left invariant symplectic structures) of these subalgebras. We first prove the following proposition, by using the results of Dynkin [6].

Proposition 5. The maximum dimension of a proper subalgebra of $\mathfrak{s l}(n, \mathbf{R})$ is equal to $n(n-1)$. And in the case $n \geq 3$, any $n(n-1)$-dimensional subalgebra of $\mathfrak{s l}(n, \mathbf{R})$ is conjugate to

or

$$
\left.\mathfrak{g}_{1}=\left\{\begin{array}{cc}
A & v \\
0 & -\operatorname{Tr} A
\end{array}\right) \mid \begin{array}{l}
A \in \mathfrak{g l}(n-1, \mathbf{R}) \\
v \in \mathbf{R}^{n-1}
\end{array}\right\}
$$

$$
\mathfrak{g}_{2}=\left\{\begin{array}{cc}
\left(\begin{array}{cc}
A & 0 \\
{ }^{t} v & -\operatorname{Tr} A
\end{array}\right) \mid \begin{array}{l}
A \in \mathfrak{g l}(n-1, \mathbf{R}) \\
v \in \mathbf{R}^{n-1}
\end{array}
\end{array}\right\}
$$

under the adjoint action of $S L(n, \mathbf{R})$. These two subalgebras are not conjugate by this action, but are mapped to each other by the outer automorphism $X \mapsto-{ }^{t} X$. In the case $n=2$, any 2-dimensional subalgebra of $\mathfrak{s l}(2, \mathbf{R})$ is conjugate to the above $\mathfrak{g}_{1}$. These subalgebras are all isomorphic to the affine Lie algebra $\mathfrak{a}(n-1, \mathbf{R})$ $(n \geq 2)$ and admit left invariant symplectic structures.

Proof. In [6], Dynkin classified maximal subgroups of classical complex simple Lie groups. From his results, we know the following facts: There are three types of maximal complex subalgebras of the Lie algebra $\mathfrak{s l}(n, \mathbf{C})$ :

(a) reducible maximal subalgebras,

(b) irreducible non-simple maximal subalgebras,

(c) irreducible simple maximal subalgebras.

From Theorem 1.1 in [6, p.252], it is easy to see that the largest dimension of the subalgebras of type (a) is $n(n-1)$, and these subalgebras admit a 1- or $(n-1)$ dimensional invariant complex subspace of $\mathbf{C}^{n}$.

It is also easy to see that the dimensions of the subalgebras of type (b) (6. p.253]) are smaller than $n(n-1)$. As for the subalgebras of type (c), we can see that the dimensions of these subalgebras are smaller than $n(n-1)$ by checking low dimensional irreducible representations of $\mathfrak{s l}(k+1, \mathbf{C}), \mathfrak{o}(4 k+2, \mathbf{C})$ and the exceptional Lie algebra $E_{6}$. (See [6], p.253-255].) Combining these results, it follows that the maximum dimension of proper complex subalgebras of $\mathfrak{s l}(n, \mathbf{C})$ is $n(n-1)$, and these subalgebras admit a 1- or $(n-1)$-dimensional invariant subspace.

Now, assume $n \geq 3$, and let $\mathfrak{g}^{\prime}$ be an $n(n-1)$-dimensional real subalgebra of $\mathfrak{s l}(n, \mathbf{R})$. (A real subalgebra of this dimension actually exists.) Then from the above fact, $\mathfrak{g}^{\prime}$ is the maximum dimensional subalgebra and the complexification $\mathfrak{g}^{\prime \mathbf{C}}$ admits a 1- or $(n-1)$-dimensional invariant complex subspace of $\mathbf{C}^{n}$. Then 
by an elementary argument, it follows that the action of $\mathfrak{g}^{\prime}$ on $\mathbf{R}^{n}$ is reducible. By using the assumption $\operatorname{dim}_{\mathbf{R}} \mathfrak{g}^{\prime}=n(n-1)$, we can easily see that $\mathfrak{g}^{\prime}$ must be expressed in the form $\mathfrak{g}_{1}$ or $\mathfrak{g}_{2}$ in Proposition 5 if we suitably change a basis of $\mathbf{R}^{n}$.

The verification of the statement for $\mathfrak{s l}(2, \mathbf{R})$ is straightforward, and the remaining statements in Proposition 5 are almost trivial. For example, the subalgebra $\mathfrak{g}_{1}$ is isomorphic to $\mathfrak{a}(n-1, \mathbf{R})$ by the isomorphism

$$
f: \mathfrak{g}_{1} \ni\left(\begin{array}{cc}
A & v \\
0 & -\operatorname{Tr} A
\end{array}\right) \longmapsto\left(\begin{array}{cc}
A+\operatorname{Tr} A \cdot I_{n-1} & v \\
0 & 0
\end{array}\right) \in \mathfrak{a}(n-1, \mathbf{R}) .
$$

Remark. (1) In the case $n=2$, the automorphism $X \mapsto-{ }^{t} X$ coincides with the inner automorphism Ad $\left(\begin{array}{cc}0 & 1 \\ -1 & 0\end{array}\right)$. The typical outer automorphism of $\mathfrak{s} r(2, \mathbf{R})$ is given by $\operatorname{Ad}\left(\begin{array}{cc}1 & 0 \\ 0 & -1\end{array}\right)$.

(2) In general, in contrast with $S L(n, \mathbf{R})$, not all maximum even dimensional subalgebras of $\mathfrak{g}$ admit a left invariant symplectic structure. For example, the Lie algebra $\mathfrak{o}(4,1) \simeq \mathfrak{s p}(1,1)$ admits several 6 -dimensional subalgebras. But, we can show that they never possess left invariant symplectic structures, and the maximum of $\operatorname{rank} \pi$ is 4 for this Lie algebra. (See [1].)

Proof of Theorem 3. On account of Proposition 5, it suffices to determine the number of equivalence classes of left invariant symplectic structures on $\mathfrak{g}_{1}$ for $n \geq 2$. We first consider the adjoint action. By an easy calculation, we can show that the subgroup of Ad $S L(n, \mathbf{R})$ which preserves $\mathfrak{g}_{1}$ is expressed as Ad $G_{1}$ where

$$
G_{1}=\left\{\left(\begin{array}{cc}
X & w \\
0 & |X|^{-1}
\end{array}\right) \mid \begin{array}{l}
X \in G L(n-1, \mathbf{R}) \\
w \in \mathbf{R}^{n-1}
\end{array}\right\} .
$$

And we have the following commutative diagram:

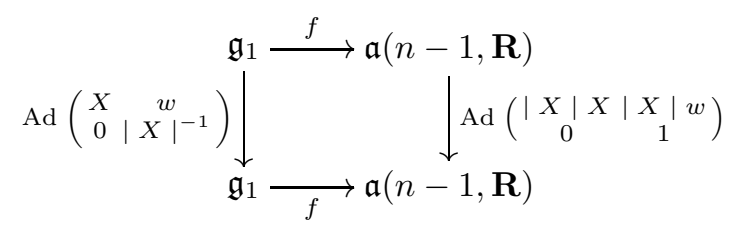

where $f$ is the isomorphism given at the end of the proof of Proposition 5. We here put

$$
\operatorname{Ad} G_{2}=\left\{\operatorname{Ad}\left(\begin{array}{cc}
|X| X & |X| w \\
0 & 1
\end{array}\right) \mid \begin{array}{l}
X \in G L(n-1, \mathbf{R}) \\
w \in \mathbf{R}^{n-1}
\end{array}\right\}
$$

Then, in case $n=o d d$, it is easy to check that the group $\operatorname{Ad} G_{2}$ just coincides with Ad $A(n-1, \mathbf{R})$. Hence, from the above diagram and from Theorem 1 , it follows that the left invariant symplectic structure on $\mathfrak{g}_{1}$ is unique under the action of $\operatorname{Ad} G_{1}$.

In the case $n=$ even, we need more delicate arguments. In this case, the above group $\operatorname{Ad} G_{2}$ coincides with the proper subgroup of $\operatorname{Ad} A(n-1, \mathbf{R})$ defined by

$$
\operatorname{Ad} A^{+}(n-1, \mathbf{R})=\left\{\operatorname{Ad}\left(\begin{array}{cc}
P & v \\
0 & 1
\end{array}\right)|| P \mid>0\right\} \subset \operatorname{Ad} A(n-1, \mathbf{R}) .
$$

It can be directly verified that the kernel of the differential $d: \mathfrak{a}(n-1, \mathbf{R})^{*} \rightarrow$ $\wedge^{2} \mathfrak{a}(n-1, \mathbf{R})^{*}$ is spanned by the 1 -form $\beta=E_{11}^{*}+\cdots+E_{n-1, n-1}^{*}$. Hence, the equality $(\operatorname{Ad} g)^{*} d \alpha=d \alpha^{\prime}\left(g \in A(n-1, \mathbf{R}), \alpha, \alpha^{\prime} \in \mathfrak{a}(n-1, \mathbf{R})^{*}\right)$ holds if and 
only if $(\operatorname{Ad} g)^{*} \alpha=\alpha^{\prime}+k \beta$ for some $k \in \mathbf{R}$. We define two non-degenerate 1-forms $\alpha_{0}, \alpha_{1} \in \mathfrak{a}(n-1, \mathbf{R})^{*}$ by

$$
\begin{aligned}
& \alpha_{0}=E_{12}^{*}+E_{23}^{*}+\cdots+E_{n-2, n-1}^{*}+E_{n-1, n}^{*}, \\
& \alpha_{1}=E_{12}^{*}+E_{23}^{*}+\cdots+E_{n-2, n-1}^{*}-E_{n-1, n}^{*} .
\end{aligned}
$$

Then, $-d \alpha_{0}$ and $-d \alpha_{1}$ give inequivalent left invariant symplectic structures on $\mathfrak{a}(n-1, \mathbf{R})$ under the action of $\mathrm{Ad} A^{+}(n-1, \mathbf{R})$. In fact, an element $g \in A(n-1, \mathbf{R})$ satisfying $(\operatorname{Ad} g)^{*} \alpha_{0}=\alpha_{1}+k \beta$ is uniquely determined (cf. Remark (2) at the end of section 2), and it is given by $g_{\alpha_{1}+k \beta}$ constructed in section 2 . We can easily see that the determinant of $g_{\alpha_{1}+k \beta}$ is equal to $(-1)^{n-1}=-1$, and hence $\alpha_{0}$ cannot be mapped to $\alpha_{1}+k \beta$ by any element of $\operatorname{Ad} A^{+}(n-1, \mathbf{R})$. In addition, any non-degenerate $\alpha \in \mathfrak{a}(n-1, \mathbf{R})^{*}$ can be mapped to $\alpha_{0}$ or $\alpha_{1}$ by $\operatorname{Ad} A^{+}(n-1, \mathbf{R})$ according to the sign of $\left|g_{\alpha}\right|$, and hence in the case $n=$ even, the subalgebra $\mathfrak{g}_{1}$ admits two left invariant symplectic structures under the action of $\mathrm{Ad} G_{1}$.

Next, we consider the outer automorphism Ad $\left(\begin{array}{ll}I_{n-1} & \\ & -1\end{array}\right)$. This automorphism preserves the subspace $\mathfrak{g}_{1}$ and commutes with the isomorphism $f$. In addition, it satisfies the equality

$$
\left(\operatorname{Ad}\left(\begin{array}{ll}
I_{n-1} & \\
& -1
\end{array}\right)\right)^{*} \alpha_{0}=\alpha_{1},
$$

and hence, a left invariant symplectic structure on $\mathfrak{g}_{1}$ is uniquely determined under the action of the automorphism group. Therefore, combining with Proposition 5, we complete the proof of Theorem 3 .

Remark. The representatives of the corresponding Poisson tensors $\pi \in \wedge^{2} \mathfrak{s l}(n, \mathbf{R})$ with $\operatorname{rank} \pi=n(n-1)$ are explicitly given as follows (cf. [1]). We put

$$
\begin{aligned}
& \pi_{1}=\sum_{1 \leq i \leq j \leq k \leq n-1}\left(E_{j i}-1 / n \cdot \delta_{j i} \cdot I_{n}\right) \wedge E_{k-j+i, k+1}, \\
& \pi_{1}^{\prime}=\sum_{1 \leq i \leq j \leq k \leq n-1} \varepsilon_{k}\left(E_{j i}-1 / n \cdot \delta_{j i} \cdot I_{n}\right) \wedge E_{k-j+i, k+1}, \\
& \pi_{2}=\sum_{1 \leq i \leq j \leq k \leq n-1}\left(E_{i j}-1 / n \cdot \delta_{i j} \cdot I_{n}\right) \wedge E_{k+1, k-j+i}, \\
& \pi_{2}^{\prime}=\sum_{1 \leq i \leq j \leq k \leq n-1} \varepsilon_{k}\left(E_{i j}-1 / n \cdot \delta_{i j} \cdot I_{n}\right) \wedge E_{k+1, k-j+i},
\end{aligned}
$$

where $\varepsilon_{k}=1$ in case $k \neq n-1$, and $\varepsilon_{n-1}=-1$. Then, under the adjoint action, the representatives of the equivalence classes are given by

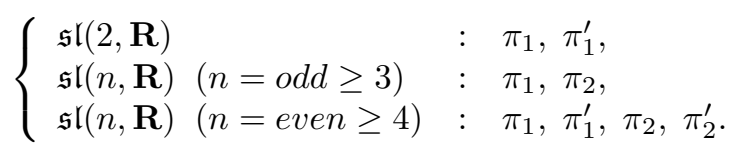

In the case of $n=2$, the set of solutions of the classical Yang-Baxter equation $[\pi, \pi]_{S}=0$ constitutes a quadratic cone in $\wedge^{2} \mathfrak{s t}(2, \mathbf{R}) \simeq \mathbf{R}^{3}$, and there are three Ad $S L(2, \mathbf{R})$-orbits represented by $\pi_{1}, \pi_{1}^{\prime}\left(=-\pi_{1}\right)$ and 0 . But for general $S L(n, \mathbf{R})$ $(n \geq 3)$, the complete orbit decomposition (= Ad $S L(n, \mathbf{R})$-classification) of the solutions of $[\pi, \pi]_{S}=0$ is unknown.

In the complex case, Stolin [13] classified the solutions of the classical YangBaxter equation for two Lie algebras $\mathfrak{s l}(2, \mathbf{C})$ and $\mathfrak{s l}(3, \mathbf{C})$ under the action of the automorphism groups of $\mathfrak{s l}(n, \mathbf{C})$. In particular, he showed that the solution with rank $\pi=n(n-1)$ is uniquely determined under this equivalence for $n=2,3$. By complexifing our arguments in the proofs of Proposition 5 and Theorem 3, we can immediately generalize Stolin's results to the following form. 
Theorem 6. The solution of the classical Yang-Baxter equation for $S L(n, \mathbf{C})$ with rank $\pi=n(n-1)$ is of maximum rank. And it is uniquely determined for $n \geq 2$ under the action of the automorphism group of $\mathfrak{s l}(n, \mathbf{C})$. The representative is given by the above $\pi_{1}$.

\section{REFERENCES}

[1] Y. Agaoka, Left invariant Poisson structures on classical non-compact simple Lie groups, Israel J. Math. 116 (2000), 189-222.

[2] A. A. Belavin and V. G. Drinfel'd, Solutions of the classical Yang-Baxter equation for simple Lie algebras, Func. Anal. Appl. 16 (1982), 159-180. MR 84e:81034

[3] M. Bordemann, A. Medina and A. Ouadfel, Le groupe affine comme variété symplectique, Tôhoku Math. J. 45 (1993), 423-436. MR 95b:53060

[4] M. Cahen, S. Gutt and J. Rawnsley, Some remarks on the classification of Poisson Lie groups, Contemporary Math. 179 (1994), 1-16. MR 96b:58031

[5] J. M. Dardié and A. Medina, Double extension symplectique d'un groupe de Lie symplectique, Adv. in Math. 117 (1996), 208-227. MR 97e:58076

[6] E. B. Dynkin, The maximal subgroups of the classical groups, Amer. Math. Soc. Translation Ser.2, Vol. 6 (1957), 245-378.

[7] Z. J. Liu and P. Xu, On quadratic Poisson structures, Lett. Math. Phys. 26 (1992), 33-42. MR 93k:58097

[8] A. Medina and P. Revoy, Groupes de Lie à structure symplectique invariante, in "Symplectic Geometry, Groupoids, and Integrable Systems" (P. Dazord, A. Weinstein, ed.), Math. Sci. Res. Inst. Publ. 20, 247-266, Springer-Verlag, New York, Berlin, 1991. MR 92k:58086

[9] K. Mikami, Symplectic and Poisson structures on some loop groups, Contemporary Math. 179 (1994), 173-192. MR 96d:58048

[10] A. I. Ooms, On Lie algebras having a primitive universal enveloping algebra, J. Algebra 32 (1974), 488-500. MR 52:8208

[11] A. I. Ooms, On Frobenius Lie algebras, Comm. in Algebra 8 (1) (1980), 13-52. MR 81c:17011

[12] M. Sato and T. Kimura, A classification of irreducible prehomogeneous vector spaces and their relative invariants, Nagoya Math. J. 65 (1977), 1-155. MR 55:3341

[13] A. Stolin, Constant solutions of Yang-Baxter equation for $\mathfrak{s l}(2)$ and $\mathfrak{s l}(3)$, Math. Scand. 69 (1991), 81-88. MR 93b:17053

[14] I. Vaisman, Lectures on the Geometry of Poisson Manifolds, Progress in Math. 118, Birkhäuser, Basel, 1994. MR 95h:58057

Department of Mathematics, Faculty of Integrated Arts and Sciences, Hiroshima University, Higashi-Hiroshima 739-8521, Japan

E-mail address: agaoka@mis.hiroshima-u.ac.jp 